\title{
PREPARAÇÃo E CARACTERIZAÇÃo ÓPTICA DE FILMES DE POLI(ESTIRENO SULFONADOS) DOPADOS COM NEODÍMIO
}

\author{
Fernando H. Cristovan, Sebastião P. Eiras e Welington O. Cruz \\ Instituto de Química, Universidade Federal de Uberlândia, CP 593, 38400-902 Uberlândia - MG \\ Alexandre Marletta* \\ Faculdade de Física, Universidade Federal de Uberlândia, CP 593, 38400-902 Uberlândia - MG \\ Debora T. Balogh \\ Instituto de Física de São Carlos, Universidade de São Paulo, CP 369, 13660-970 São Carlos - SP \\ Edson Laureto, José L. Duarte e Ivan F. L. Dias \\ Departamento de Física, Universidade Estadual de Londrina, CP 6001, 86051-990 Londrina - PR
}

Recebido em 28/6/04; aceito em 18/3/05; publicado na web em 2/8/05

\begin{abstract}
PROCESSING AND OPTICAL CHARACTERIZATION OF POLY(STYRENE SULPHONATE) FILMS DOPED WITH NEODYMIUM. In this work we describe the processing of poly(styrene sulphonate) films (PSS) doped with neodymium (Nd). Optical density measurements in the UV-Vis-NIR region show the typical bands observed for neodymium chloride $\left(\mathrm{NdCl}_{3}\right)$ in solution. In the case of films, the intensity ratio between the peaks at $800 \mathrm{~nm}\left({ }^{4} \mathrm{I}_{9 / 2} \rightarrow{ }^{4} \mathrm{~F}_{5 / 2}+{ }^{2} \mathrm{H}_{7 / 2}\right)$ and $580 \mathrm{~nm}\left({ }^{4} \mathrm{I}_{9 / 2} \rightarrow{ }^{4} \mathrm{G}_{5 / 2}+{ }^{2} \mathrm{G}_{7 / 2}\right)$ is equal to 0.83 . Infrared spectra present an enhancement in the absorption region of aromatic rings. Site selective luminescence spectroscopy shows that the incorporation of $\mathrm{Nd}$ introduces a hipsochromic shift and a line shape definition in UV luminescence compared to PSS film, decreasing the interaction between aromatic groups. In addition, the film exhibits an intense radiative transition at $1061 \mathrm{~nm}\left({ }^{4} \mathrm{~F}_{3 / 2} \rightarrow{ }^{4} \mathrm{I}_{11 / 2}\right)$, comparable to the one present in crystalline materials doped with $\mathrm{Nd}$.
\end{abstract}

Keywords: poly(styrene sulphonate); neodymium; films.

\section{INTRODUÇÃO}

O estudo de novos materiais utilizando Terras-Raras (TRs) tem sido explorado nos últimos anos devido ao crescente interesse, principalmente, nas possíveis aplicações tecnológicas em dispositivos fotônicos. Neste caso, os TRs são largamente utilizados, desde a produção de componentes ópticos, vidros e lâmpadas fluorescentes até equipamentos de energia nuclear, estando presentes em eletrodomésticos (refrigeradores), discos rígidos (HDs) de computadores pessoais, catalisadores e muitos outros produtos produzidos por empresas de alta tecnologia ${ }^{1,2}$. Além disso, os lantanídeos (Ln) são de grande interesse científico, uma vez que suas propriedades ópticas não dependem muito do material hospedeiro. Isto se deve a sua estrutura eletrônica, na qual os orbitais mais externos, $5 \mathrm{~d}$ e $6 \mathrm{~s}$, são responsáveis pelas ligações químicas, blindando o orbital 4f que está semipreenchido ${ }^{3}$. Em particular, as propriedades ópticas dos íons de TRs, denominados $\mathrm{Ln}^{3+}$, devem-se às fracas interações entre os elétrons do nível 4f e o campo cristalino no qual está inserido. O resultado final é a observação de transições eletrônicas bem definidas, associadas a níveis eletrônicos atômicos.

No contexto deste trabalho, o interesse pelo íon $\mathrm{Nd}^{3+}$ deve-se ao fato deste elemento possuir uma configuração eletrônica envolvendo o nível 4f com transições de alta eficiência quântica e emissão de luz aproximadamente em 1060 nm (atribuída à transição $\left.{ }^{4} \mathrm{~F}_{3 / 2} \rightarrow{ }^{4} \mathrm{I}_{11 / 2}\right)^{4}$. Devido a isso, o neodímio é considerado um dos elementos de maior interesse na produção de materiais aplicados à fabricação de dispositivos para emissão laser. Outra característica importante deste material é sua distribuição simplificada de níveis (quatro níveis atômicos), o que facilita em muito a inversão de população ${ }^{4}$.

Apesar do grande número de trabalhos científicos que empre-

*e-mail: marletta@ufu.br gam estes íons de TRs como dopantes em meio ativo, o uso de matrizes poliméricas como matriz hospedeira não tem sido explorado. A grande dificuldade em sua utilização está associada ao desenvolvimento de rotas sintéticas que permitam a incorporação destes íons ao longo da cadeia principal ou das cadeias laterais do polímero. No presente trabalho é apresentada uma rota sintética e o processamento de filmes de poli(estireno sulfonados) dopados com $\mathrm{Nd}^{3+}$, sua caracterização química e o estudo das suas propriedades fotofísicas.

\section{PARTE EXPERIMENTAL}

O poliestireno sulfonado (PSS) na forma sódica $\left(\mathrm{M}_{\mathrm{w}}=\right.$ $70000 \mathrm{~g} \mathrm{~mol}^{-1}$, Aldrich), foi convertido para forma ácida (H-PSS) utilizando-se a resina de troca iônica Amberlite IR-120 (Aldrich). Em seguida, em um balão de duas bocas, equipado com um agitador magnético e um "trap" para gases, contendo $0,250 \mathrm{~mL}$ de uma solução de $\mathrm{NdCl}_{3} 0,5 \mathrm{~mol} \mathrm{~L}^{-1}$ em água, foi adicionada lentamente $1,5 \mathrm{~mL}$ de uma solução de H-PSS $0,15 \mathrm{~mol} \mathrm{~L}^{-1}$. Selou-se o balão com um septo e manteve-se a mistura sob agitação durante 10 min, à temperatura ambiente, sob linha de nitrogênio. Ao término desse período deixou-se a mistura reacional em repouso por $12 \mathrm{~h}$, aproximadamente a $5{ }^{\circ} \mathrm{C}$. Decorrido este tempo observou-se a formação de um precipitado (gel). Descartou-se o sobrenadante e secouse o produto obtido em um dessecador à pressão reduzida. Esta reação é apresentada esquematicamente na Figura 1.

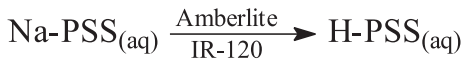

$$
\begin{aligned}
& \mathrm{H}-\mathrm{PSS}_{(\mathrm{aq})}+\mathrm{Nd}^{3+}{ }_{(\mathrm{aq})} \longrightarrow \mathrm{Nd}-\mathrm{PSS}_{(\mathrm{s})}+\mathrm{H}^{+}{ }_{(\mathrm{aq})}
\end{aligned}
$$

Figura 1. Esquema da rota de síntese química do Nd-PSS 
Os espectros de absorção na região do UV-Vis-NIR (ultravioleta ao infravermelho próximo) foram obtidos em espectrofotômetro Hach $4000 \mathrm{U}$ e os de infravermelho (IR) em um Schimadzu FTIR 8101. As medidas de emissão em função da excitação na região espectral do UV-Vis foram feitas utilizando-se um espectrofluorímetro Hitachi F-4500.

Finalmente, a medida de fotoluminescência na região do infravermelho foi efetuada utilizando-se como fonte de excitação $(514,5 \mathrm{~nm})$ um feixe não focalizado de um laser de argônio contínuo. A emissão da amostra foi analisada por monocromador JarrelAsh de 0,5 m, com rede de difração de 590 ranhuras/mm ("blaze" em $1200 \mathrm{~nm}$ ), e detectada por Fotodiodo PIN de InGaAs refrigerado termoeletricamente no modo "lock-in".

\section{RESULTADOS}

Na Figura 2a são apresentados os espectros de UV-Vis-NIR normalizados dos filmes de Nd-PSS, H-PSS e de uma solução aquosa de $\mathrm{NdCl}_{3}$, indicando-se as transições eletrônicas do $\mathrm{Nd}^{3+}$. O diagrama de energia e as transições permitidas para a absorção do $\mathrm{Nd}^{3+}$ são mostrados esquematicamente na Figura 2b. Inicialmente observa-se que o filme H-PSS é transparente na região de comprimento de onda do UV-Vis-Nir. Em solução aquosa este polímero mostra um pico em $265 \mathrm{~nm}$, não apresentado aqui, referente às transições $\pi-\pi^{*}$ do anel aromático presentes nos grupos laterais do PSS $^{5}$. Observa-se ainda, um aumento da intensidade de absorção abaixo de $400 \mathrm{~nm}$, possivelmente devido ao espalhamento de luz causado pela não homogeneidade do filme e/ou absorção de novos estados eletrônicos, tal como excímeros, devido ao aumento da interação entre-cadeias poliméricas ${ }^{5}$.

Comparativamente, os espectros do $\mathrm{NdCl}_{3}$ e do Nd-PSS apresentam as mesmas transições eletrônicas com um deslocamento batocrômico de $5 \mathrm{~nm}$ para o último caso. Abaixo de $400 \mathrm{~nm}$ é observado um aumento da intensidade de absorção para o $\mathrm{Nd}$ PSS, devido ao espalhamento de luz provocado pela matriz de $\mathrm{H}$ PSS. No filme de Nd-PSS, a razão entre as intensidades nos picos

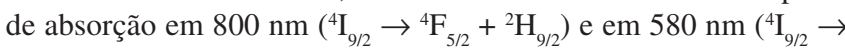
${ }^{4} \mathrm{G}_{5 / 2}+{ }^{2} \mathrm{G}_{7 / 2}$ ) é igual a 1,2 ; no caso do cloreto de neodímio dissolvido em água, esta razão é 1,5. Na transição de mais alta energia, a banda em $580 \mathrm{~nm}$ é mais susceptível às mudanças do ambiente químico ao redor do íon TR, sendo assim hipersensível e dependente da matriz ${ }^{6}$. Vidros dopados com neodímio, geralmente, apresentam bandas mais alargadas em relação ao $\mathrm{NdCl}_{3}$ e absorção em $590 \mathrm{~nm}$ mais intensa, chegando a ser 1,6 vez maior que a em $800 \mathrm{~nm}^{6}$.

A verificação da incorporação do $\mathrm{Nd}^{3+}$ na matriz polimérica, além dos dados de absorção apresentados na Figura 2, foi obtida analisando-se os espectros de IR dos filmes H-PSS e Nd-PSS, apresentados na Figura 3. Nesta figura são observadas as bandas de absorção características do H-PSS ${ }^{7-9}$, a saber estiramento simétrico da ligação $\mathrm{S}=\mathrm{O}$ em $1190 \mathrm{~cm}^{-1}$, estiramentos simétricos das ligações O-S-O de 1000 a $1030 \mathrm{~cm}^{-1}$, deformação angular fora do plano do $\mathrm{C}-\mathrm{H}$ dos anéis aromáticos em $800 \mathrm{~cm}^{-1}$ e estiramento simétrico do O-H entre 3900 e $3000 \mathrm{~cm}^{-1}$. O Nd-PSS apresenta um espectro semelhante ao do H-PSS, observando-se apenas um aparente alargamento em toda sua extensão. Isto indica, indiretamente, a incorporação do $\mathrm{Nd}^{3+}$, como mostrado por Sun e colaboradores ${ }^{10}$ em complexos de poli(bitiazol-tetratiapentaleno) dopados com neodímio. Estes autores verificaram o mesmo alargamento no espectro de IR e atribuíram este efeito à coordenação do $\mathrm{Nd}^{3+}$ com o $\mathrm{S}$ e o $\mathrm{N}$ do polímero. De maneira análoga, para os filmes de $\mathrm{Nd}$ PSS pode-se considerar que ocorreu a coordenação do neodímio com os grupos sulfônicos do H-PSS.
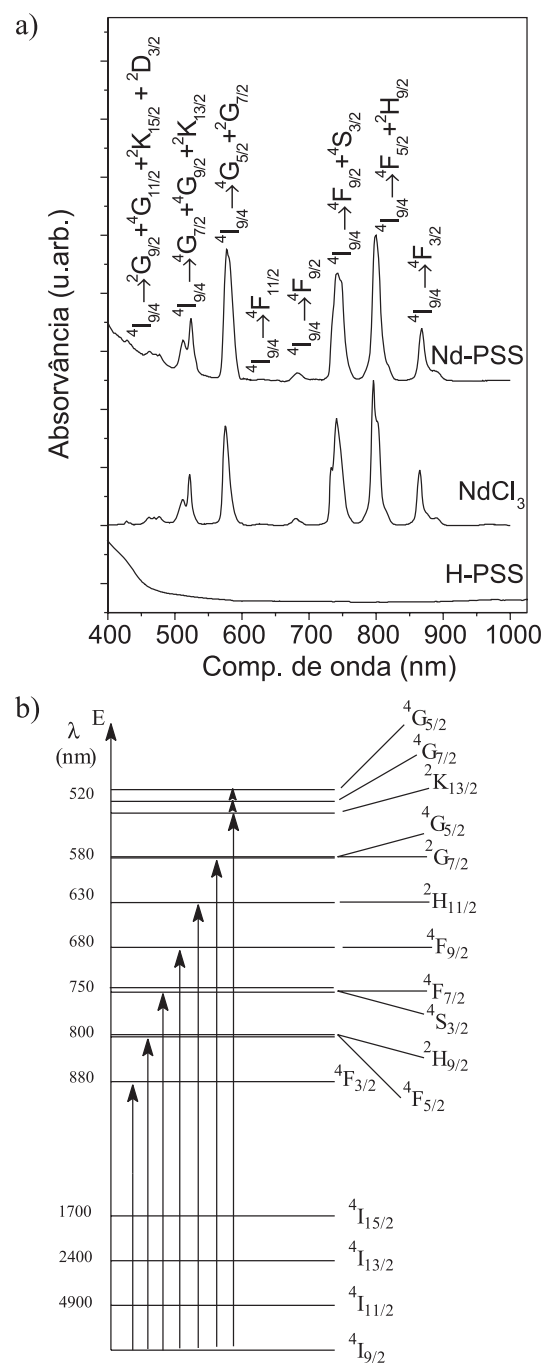

Figura 2. a) Espectro de absorção no UV-Vis-NIR de um filme de Nd-PSS contendo $13,92 \% \mathrm{~mol} / \mathrm{mol}$ de $\mathrm{Nd}^{3+}$ e do $\mathrm{NdCl}_{3}, 20 \times 10^{-3} \mathrm{~mol} \mathrm{~L}^{-1}$ em solução aquosa; (b) diagrama de energia para as transições eletrônicas do neodímio na matriz de poliestireno sulfonado

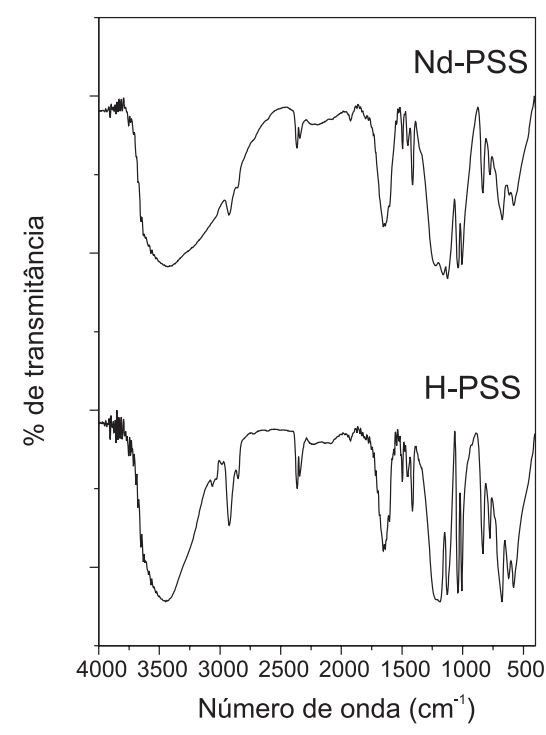

Figura 3. Espectros de IR do Nd-PSS e do H-PSS 
Na Figura 4 são mostrados os espectros de emissão normalizados do H-PSS (Figura 4a) e Nd-PSS (Figura 4b) em função do comprimento de onda de excitação, à temperatura ambiente. No caso do H-PSS verifica-se uma banda intensa na região espectral entre 350 e $650 \mathrm{~nm}$ localizada na região de absorção, como apresentado na Figura 2. Este resultado corrobora o fato de que, geralmente, em estado sólido a interação intermolecular é mais intensa com a formação de excímeros, deslocando o espectro de emissão para o vermelho em relação ao H-PSS em solução aquo$\mathrm{sa}^{5}$. Para uma excitação com energia menor (375 e $400 \mathrm{~nm}$ ), o espectro de emissão apresenta um deslocamento para o vermelho com uma maior definição dos picos. A inclusão do $\mathrm{Nd}^{3+}$ produz efetivamente dois efeitos: deslocamento hipsocrômico de $50 \mathrm{~nm}$ da emissão do poli(estireno sulfonado) e um estreitamento das linhas de emissão. Estes dados indicam que a inclusão do $\mathrm{Nd}(\mathrm{III})$ na matriz diminui a interação dos grupos aromáticos, mostrando claramente que o espectro do H-PSS é formado por três bandas de emissão. Podemos supor que estas emissões estejam associadas à formação de excímeros ${ }^{5}$. Finalmente, a inclusão do neodímio ocorre de modo a propiciar a formação de um complexo metáli-
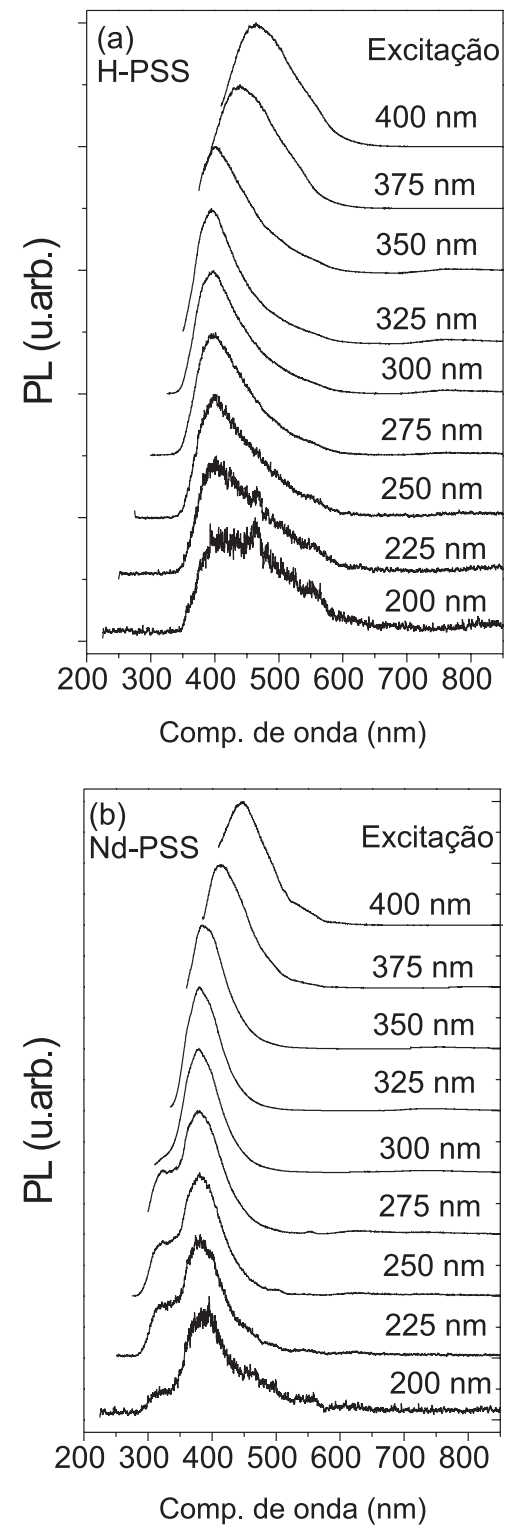

Figura 4. Espectros de emissão dos filmes H-PSS (a) e Nd-PSS (b) em função do comprimento de onda de excitação de 200 a $400 \mathrm{~nm}$ co, ligando-se a dois ou três grupos $\mathrm{SO}_{3}{ }^{-}$dos monômeros do PSS. Este fato foi também observado por Sun e colaboradores ${ }^{10}$ com o polímero bitiazol-tetratiapentaleno.

As transições radiativas na região do infravermelho, de interesse para a emissão laser deste material, são apresentadas na Figura 5. Observam-se três bandas características do $\mathrm{Nd}^{3+}$, tal como em sistemas amorfos (vidros) e cristalinos ${ }^{11}$. No detalhe desta figura apresenta-se um diagrama de energia para excitação e emissão do filme. A presença dos grupos O-H (Figura 2) pode suprimir a luminescência do $\mathrm{Nd}^{3+}$ devido à absorção de fônons com energia entre 3000 a $4000 \mathrm{~cm}^{-1}$, que é da ordem de $1 / 3 \mathrm{da}$ energia de emissão da transição ${ }^{4} \mathrm{~F}_{3 / 2} \rightarrow{ }^{4} \mathrm{I}_{11 / 2}\left(9488 \mathrm{~cm}^{-1}\right)^{12,13}$. Entretanto, para o Nd-PSS este efeito não é significativo, observando-se uma emissão intensa em 1061 nm (Figura 5) se refere à transição eletrônica ${ }^{4} \mathrm{~F}_{3 / 2} \rightarrow{ }^{4} \mathrm{I}_{11 / 2}$ do neodímio. Comparativamente com materiais cristalinos ${ }^{11}$, esta transição também é, aproximadamente, cerca de 10 vezes mais intensa que as transições ${ }^{4} \mathrm{~F}_{3 / 2} \rightarrow{ }^{4} \mathrm{I}_{13 / 2}(887 \mathrm{~nm})$ e ${ }^{4} \mathrm{~F}_{3 / 2} \rightarrow{ }^{4} \mathrm{I}_{9 / 2}(1324 \mathrm{~nm})$. No caso de outros sistemas poliméricos dopados com íon $\mathrm{Nd}$ esta razão decresce para menos que 5 vezes ${ }^{14,15}$.

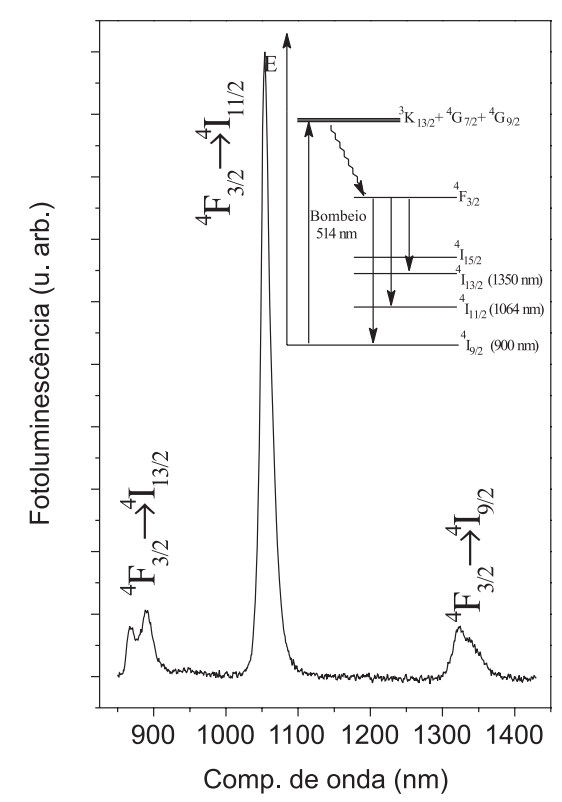

Figura 5. Espectros de emissão do Nd-PSS na região do infravermelho

\section{CONCLUSÕES}

Neste trabalho foram estudadas a preparação e a caracterização óptica de filmes de poliestireno sulfonados dopados com íons de neodímio Nd-PSS. O estudo inicial da síntese e caracterização óptica deste filme, utilizando-se como material hospedeiro o H-PSS e o dopante $\mathrm{Nd}(\mathrm{III})$, indica que os filmes são formados por processos de complexação, do tipo metálica. Uma vantagem dos filmes de Nd-PSS sobre, por ex., vidros dopados com Nd à base de aluminato é a de uma absorção mais intensa em $800 \mathrm{~nm}$ $\left({ }^{4} \mathrm{I}_{9 / 2} \rightarrow{ }^{4} \mathrm{~F}_{5 / 2}+{ }^{2} \mathrm{H}_{9 / 2}\right)$. Esta região é de grande interesse por ser facilmente excitada com o emprego de diodos lasers e pela menor probabilidade de perdas de energia por decaimentos não radiativos via fônons. Outra vantagem é que a presença do $\mathrm{Nd}$ reduz as interações entre cadeias poliméricas, diminuindo a formação de excímeros no PSS. A influência de grupos remanescentes O-H da matriz polimérica não é significativa para a supressão da emissão em $1061 \mathrm{~nm}\left({ }^{4} \mathrm{~F}_{3 / 2} \rightarrow{ }^{4} \mathrm{I}_{11 / 2}\right)$ do neodímio, mostrando 
assim que este material é bastante promissor na produção de novos materiais para emissão $\operatorname{laser}^{14}$.

\section{AGRADECIMENTOS}

Ao suporte financeiro da IMMP/MCT, FAPEMIG, FAPESP, FBB e do CNPq. Ao Prof. M. Tabak, do Instituto de Química de São Carlos da Universidade de São Paulo, por ter realizado as medidas de emissão na região do UV-Vis.

\section{REFERÊNCIAS}

1. Ronda, C. R.; Jüstel, J.; Nikol, H.; J. Alloys Compd. 1998, 227, 669.

2. Leskelã, M.; J. Alloys Compd. 1998, 227, 702.

3. Moeler, T.; The Chemistry of the Lanthanides, Reinhol Publ. Coop.: New York, 1963.

4. Hüfner, S.; Optical Spectra of Transparent Rare Earth Compounds, $2^{\text {nd }} \mathrm{ed}$., Academic Press: New York, 1978.

5. Phillips, D.; Polymer Photophysics Luminescence, Energy Migration and Molecular Motion in Synthetic Polymers, Chapman and Hall: New York, 1985.
6. Pecoraro, E.; Tese de Doutorado, Instituto de Física de São Carlos, Universidade de São Paulo, Brasil, 1999.

7. Paiva, D. L.; Lampman, G. M.; Kris, G. S.; Introduction to Spectroscopy, $2^{\text {nd }}$ ed., Saunders College: USA, 1996.

8. Pretsch, E.; Clerc, T.; Seibl, J.; Simon, W.; Tablas para la Elucidacion Estrutural de Compestos Organicos por Metodos Espectroscopicos, Ed. Alhambra: Espanha, 1980.

9. Silverstein, R. M.; Bassler, G. C.; Morrill, T. C.; Identificação Espectrométrica de Compostos Orgânicos, 5ª ed., Rio de Janeiro: Brasil, 2000.

10. Sun, W.; Jiang, L.; Weng, J.; He, B.; Cen, D.; React. Funct. Polym. 2003, $55,249$.

11. Qin, L. J.; Meng, X. L.; Zhu, L.; Liu, J. H.; Xu, B. C.; Xu, H. Z.; Jiang, F. Y.; Du, C. L.; Wang, X. Q.; Shao, Z. S.; Chem. Phys. Lett. 2003, 380, 273.

12. Pecoraro, E.; Nunes, L. A. O.; Sampaio, J. A.; Gama, S.; Baeso, M. L.; Quim. Nova 2000, 23, 161.

13. Nova, S. P. V.; Pereira, G. A. L.; Sá, G. F.; Alves Jr., S.; Bazin, H.; Autiro, H.; Mathis, G.; Quim. Nova 2004, 27, 709.

14. Lin, S.; Fereurestein, R. J.; Mickelson, A. R.; J. Appl. Phys. 1996, 79, 2868.

15. Slooff, L. H.; Polman, A.; Klink, S. I.; Hebbink, G. A.; Grave, L.; van Veggel, F. C. J. M.; Reinhoudt, D. N.; Hofstraat, J. W.; Opt. Materials 2000, $14,101$. 